\title{
Multiple Brown Tumors Caused by Primary Hyperparathyroidism as a Differential Diagnosis to Multiple Osteolytic Bone Metastases: A Case Report
}

\author{
Zeina Hadad ${ }^{\mathrm{a}, \mathrm{d}}$, Louise Tjelum ${ }^{\mathrm{a}}$, Pia Eiken ${ }^{\mathrm{a}}$, Waldemar Trolle ${ }^{\mathrm{b}}$, \\ Ilia Haupter ${ }^{\mathrm{c}}$, Pia Afzelius ${ }^{\mathrm{c}}$
}

\begin{abstract}
Brown tumors are benign osteolytic lesions, which usually respect the bone cortex. Since these lesions may resemble bone metastases, it is important to consider them as a potential differential diagnosis. They occur as a result of increased parathyroid hormone (PTH) secretion mainly due to primary or secondary hyperparathyroidism. We present a case of multiple osteolytic lesions incidentally found on X-ray examinations in a patient, who had a radius fracture after a low-energy trauma. Due to the suspicion of multiple bone metastases, one of them mimicking sequels after a pathological fracture in the ulna, the patient had a positron emission tomography/computed tomography (PET/CT) and a magnetic resonance imaging (MRI) scan performed supporting the existence of pervasive bone lesions without suggesting a primary malignancy. The blood samples showed highly elevated ionized calcium and PTH levels. Therefore, an ultrasound examination and parathyroid scintigraphy were performed, revealing a hyperfunctioning parathyroid adenoma. After removal of the adenoma, the PTH level normalised and the bone changes regressed without surgical intervention.
\end{abstract}

Keywords: Primary hyperparathyroidism; Parathyroid adenoma; Brown tumors; Metastasis; Osteolytic bone lesions

\section{Introduction}

Brown tumor is also known as osteitis fibrosa cystica and less

Manuscript submitted March 17, 2020, accepted March 26, 2020

Published online August 26, 2020

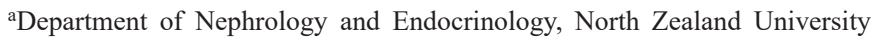
Hospital, Dyrehavevej 29, 3400 Hillerod, Denmark

bepartment of Oto-Rhino-Laryngology, North Zealand University Hospital, Dyrehavevej 29, 3400 Hillerod, Denmark

${ }^{c}$ North Zealand University Hospital, Dyrehavevej 29, 3400 Hillerod, Denmark ${ }^{\mathrm{d} C o r r e s p o n d i n g ~ A u t h o r: ~ Z e i n a ~ H a d a d, ~ D e p a r t m e n t ~ o f ~ N e p h r o l o g y ~ a n d ~ E n d o-~}$ crinology, North Zealand University Hospital, Dyrehavevej 29, 3400 Hillerod, Denmark. Email: Zeina.Hadad@aarhus.rm.dk

doi: https://doi.org/10.14740/jem637 commonly as osteoclastoma. These bone lesions arise in the setting of hyperparathyroidism (HPT) usually caused by primary hyperparathyroidism (PHPT). A parathyroid adenoma (PTA) accounts for approximately $85 \%$ of cases. Fifteen percent of PHPT cases occur due to parathyroid hyperplasia, and parathyroid carcinomas (PTC) causes less than $1 \%$ of PHPT cases [1-6]. Brown tumors may also be seen in secondary hyperparathyroidism (SHPT) [7]. The presence of brown tumors in the skeleton is a pathognomonic sign of PHPT and SHPT [8]. These tumors are rare and have an incidence of $3 \%$ and $1.5 \%$ in PHPT and SHPT, respectively [9].

The tumors are benignly caused by excessive bone resorption as the result of an over-secretion of parathyroid hormone (PTH) [2, 7, 10-13], and are often mistaken for metastases due to their osteolytic appearance $[1,10,14-16]$. The tumors are usually well-circumscribed on X-ray examination. However, in few cases, they are not respecting the bone cortex $[4,17]$. Brown tumors are reversible after normalisation of PTH $[4$, $6,12,18,19]$. In this study, we report a patient with multiple osteolytic lesions. The lesions were initially considered to represent metastases but turned out to be brown tumors as the patient had an undiagnosed PHPT. Some of the lesions did not respect the bone cortex, which is extremely rare in case of brown tumors.

\section{Case Report}

A 62-year-old previously healthy woman referred for X-ray examinations of the right elbow, pelvis and both lower extremities after a fall from standing height. The patient had a fracture of the radius head beside an incidental expansive osteolytic lesion in the ulna involving the bone cortex and mimicking sequels after pathological facture. Besides, the patient had osteolytic lesions in both femurs and the right tibia (Fig. 1).

Initially, a magnetic resonance imaging (MRI) and a positron emission tomography/computed tomography (PET/CT) scan were performed to rule out a primary malignant tumor. The MRI scan of the pelvis revealed osteolytic lesions in both femurs (Fig. 2a, b), while PET image acquired $60 \mathrm{~min}$ after injection of $272 \mathrm{MBq}$; and $\left({ }^{18} \mathrm{~F}\right) \mathrm{FDG}$ showed several foci in the skeleton accumulating the PET tracer (Fig. 2c). 

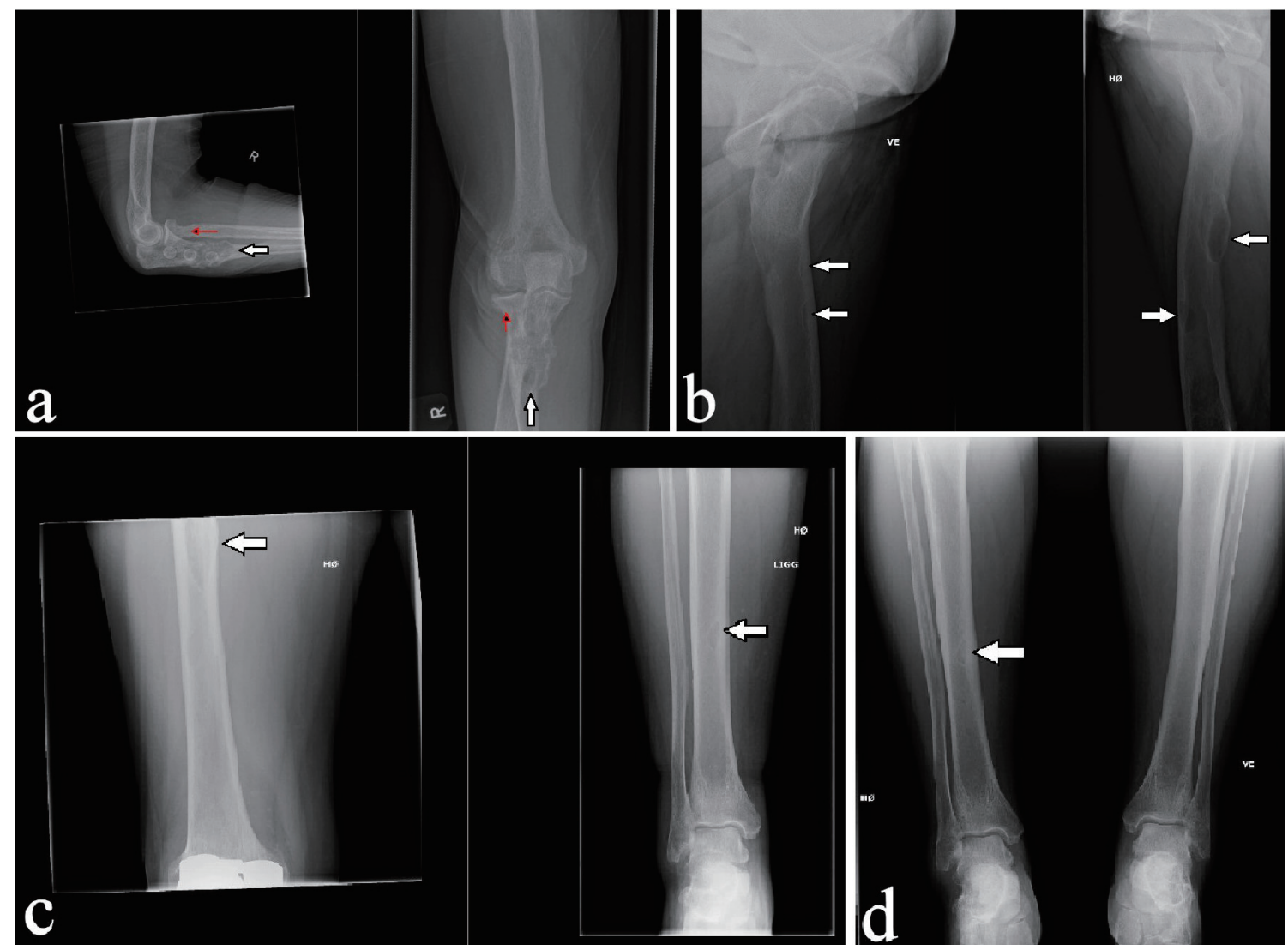

Figure 1. Initial X-ray of the right elbow showing fracture of the head of the radius (red arrow) and an expansive osteolytic lesion in the ulna mimicking sequels after pathological fracture (a) and X-rays of both lower extremities showing osteolytic lesions in both femurs (b) and right tibia (c and d) (white arrows).

Since the blood samples indicated primary PHPT, multiple brown tumors were suggested. The initial paraclinical parameters were as follow: ionized calcium $2.08 \mathrm{mmol} / \mathrm{L}(1.18-1.32)$, phosphate $0.71 \mathrm{mmol} / \mathrm{L}(0.76-1.41)$, urate $0.41 \mathrm{mmol} / \mathrm{L}(0.61$ - 0.40), creatinine $68 \mu \mathrm{mol} / \mathrm{L}(50$ - 90), 25-OH-D-vitamin 26 $\mathrm{nmol} / \mathrm{L}(>50)$, hemoglobin $6.0 \mathrm{mmol} / \mathrm{L}(7.3-9.5)$, alkaline phosphatases 744 U/L (35 - 105), PTH 369 pmol/L (1.48 7.63), and a negative M-component. Besides, the patient had nephrolithiasis. Parathyroid scintigraphy and ultrasonic sound examination demonstrated a solitary left-sided hyperfunctioning PTA (Figs. 3, 4).

The patient underwent parathyroidectomy (Fig. 5). Ten minutes after the removal of the PTA, the PTH level declined by $72 \%(56 \mathrm{pmol} / \mathrm{L})$. The subsequent histopathology confirmed a benign PTA. The PTA weighed $4.9 \mathrm{~g}$.

Preoperatively, the patient received intravenous fluid infusion for rehydration and $5 \mathrm{mg}$ zoledronic acid for treatment of hypercalcemia. After removal of the parathyroid gland, the ionized calcium level in plasma normalized over the next days. Afterwards the patient experienced an episode of hypocalcemia and developed "hungry bone syndrome" (HBS) on the fifth day postoperatively. She received high amounts of calcium carbonate, vitamin D3, active vitamin D (alfacalcidol) and magnesium. She was gradually reduced in alfacalcidol and discontinued 9 months after the operation and continued with calcium carbonate and vitamin D3.

Twelve month after removal of the PTA, a dual energy $\mathrm{X}$-ray absorption (DXA) scan showed compared to the initial DXA scan that was performed just before operation, that the bone mineral density (BMD) was increased from $0.724 \mathrm{~g} /$ $\mathrm{cm}^{2}$ (T-score: -2.9 ) to $0.987 \mathrm{~g} / \mathrm{cm}^{2}$ (T-score: -0.5 ) in the lumbar spine and from $-0.625 \mathrm{~g} / \mathrm{cm}^{2}$ (T-score: -2.0 ) to 1.016 (Tscore: +1.5$)$ in the left femoral neck. In the trabecular bone of distal one-third of left radius, the BMD increased from 0.436 $\mathrm{g} / \mathrm{cm}^{2}$ (T-score: -4.3 ) to $0.522 \mathrm{~g} / \mathrm{cm}^{2}$ (T-score: -2.9 ). After about 14 months, the lesions in the right ulna and the proximal right femur regressed considerably (Figs. 6, 7), while the other bone lesions were almost unrecognizable (Figs. 7b, 8). The patient reported no symptoms of illness before hospitalization, but retrospectively she had experienced fatigues, leg pain, increased thirst, loss of appetite, and weight loss for months.

\section{Discussion}

Brown tumors are benign lesions with osteolytic radiographic appearance often arising in multiple bones [1, 10, 14-16]. They are considered as an uncommon complication of prolonged PHPT $[3,6,9]$. These tumors contain hemosiderin, hence the 


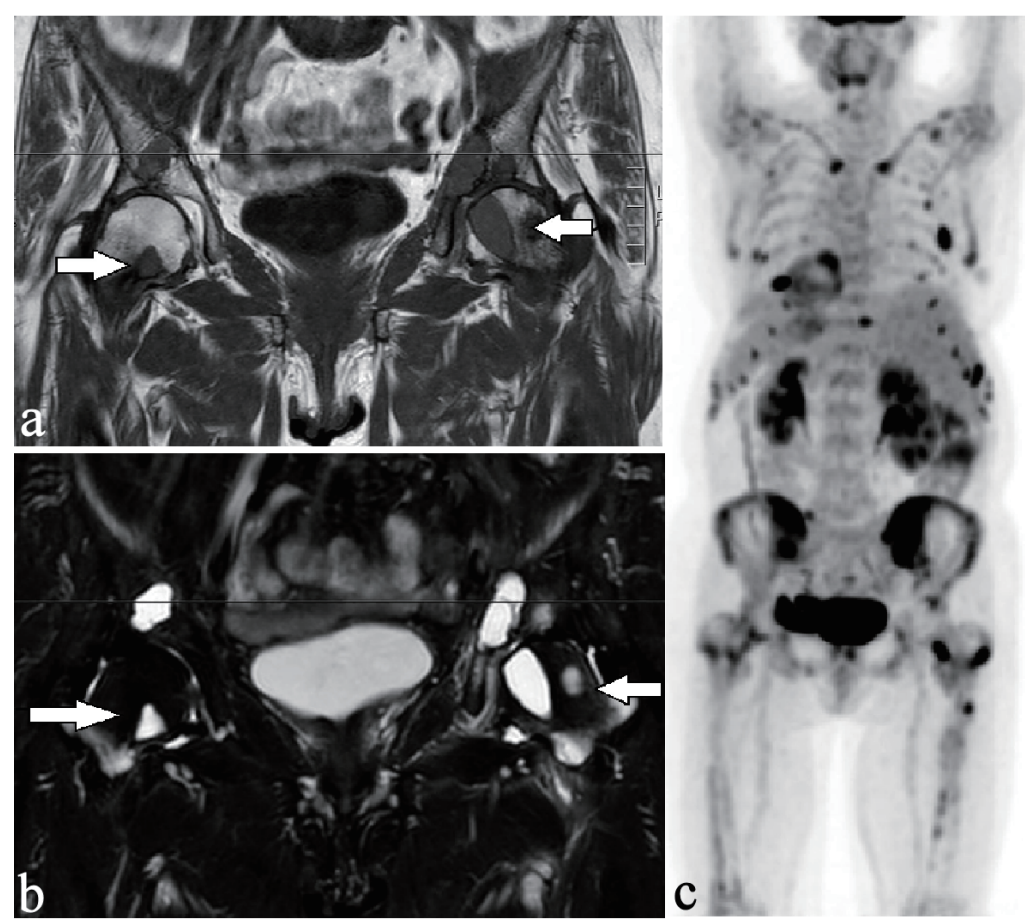

Figure 2. Osteolytic lesions had a low-intensity signal on the T1-weighted sequence (a) and had a high-intensity signal on the STIR sequence (b) in both femurs (arrows). The coronal PET scan showed high $\left({ }^{18} \mathrm{~F}\right)$ FDG metabolism in multiple osteolytic lesions in the cervical spine, both clavicles, the ribs, the pelvis, and both femurs (c). PET: positron emission tomography.

distinctive brown color $[1,6,17]$.

Brown tumors tend to appear well-circumscribed respecting the bone cortex, although in some rare cases they can have an invading appearance making them even more similar to metastases $[4,14,17]$. In our case, the osteolytic lesion in the proximal ulna on the initial X-ray examination seemed to be affecting the cortex and resembled sequels after a pathological fracture. This, along with other osteolytic lesions in the pelvis and lower extremities, caused malignancy to be mistakenly suspected.

Excessive secretion of PTH in PHPT causes enhanced os- teoclastic activity of the bones and increased calcium absorption in the renal tubules resulting in hypercalcemia [2]. This have been encountered in our case and was reported by other similar cases $[6,10-12,14,15,18]$. Parathyroidectomy is the only curative treatment in PHPT [3]. The level of PTH falls quickly after successful removal of hyperactive parathyroid tissue. A decrease in the level of PTH intraoperatively by more than $50 \%$ or normalisation of PTH level 10 min after parathyroidectomy is expected $[2,4,19]$. This steep reduction of PTH level leads to decrease of the renal tubular reabsorption of calcium. The osteoblastic activity displaces the osteoclastic activ-
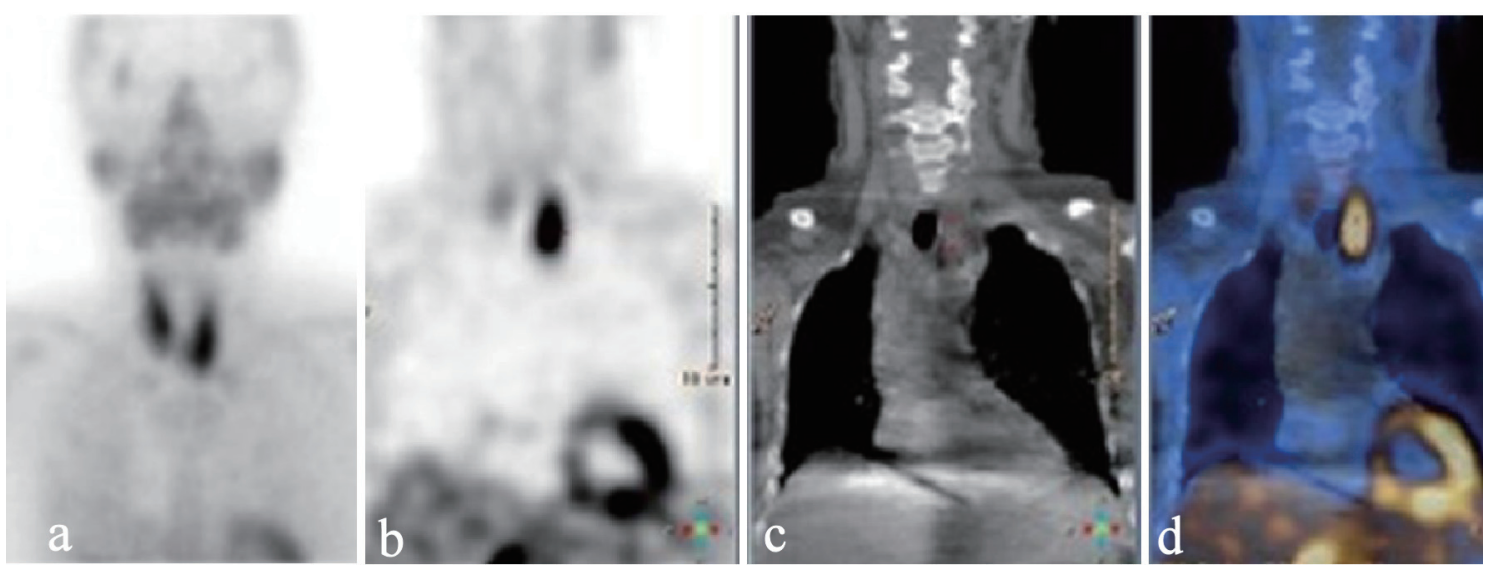

Figure 3. Static parathyroid scintigraphy after injection of ${ }^{99 \mathrm{~m} T c-p e r t e c h n e t a t e ~ i n ~ e a r l y ~ p h a s e ~(a), ~ a n d ~ l a t e ~ p h a s e ~(a f t e r ~ w a s h-o u t) ~}$ (b) showing accumulation related to the caudal part of the left thyroid lobe. Low-dose CT (c) and fused image of the scintigraphy and the low-dose CT (d) suggested a parathyroid adenoma. CT: computed tomography. 


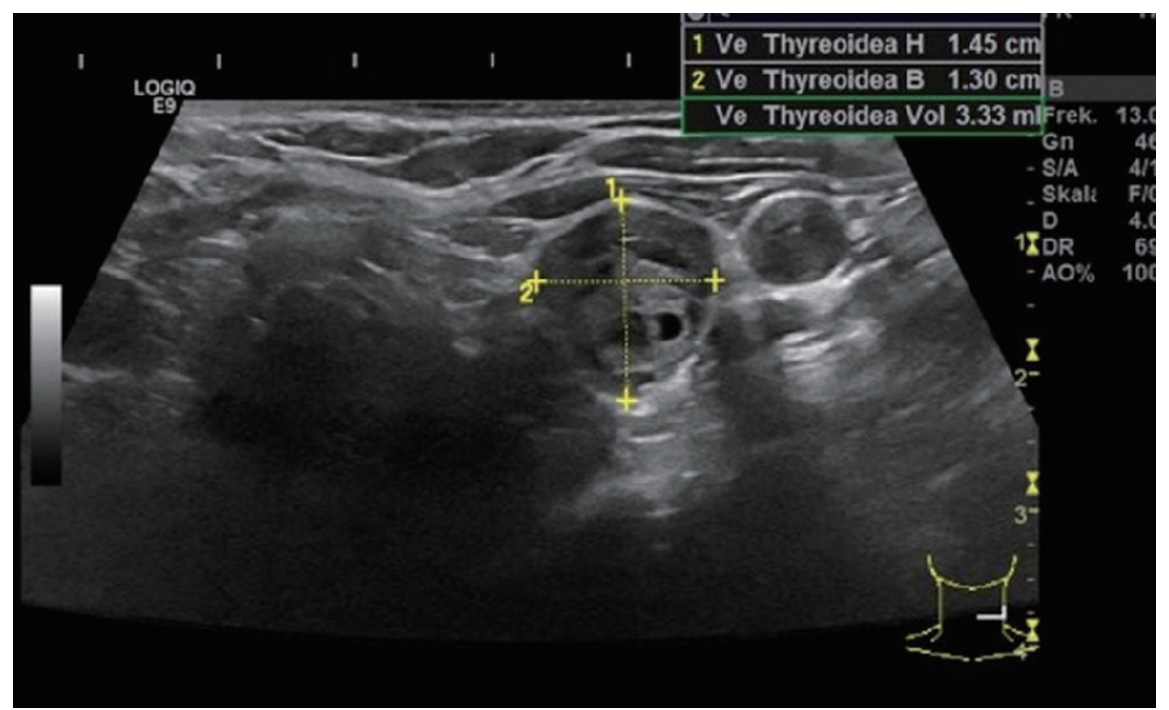

Figure 4. Ultrasound examination showing a hypoechoic adenoma that had an estimated weight of $3.3 \mathrm{~g}$.
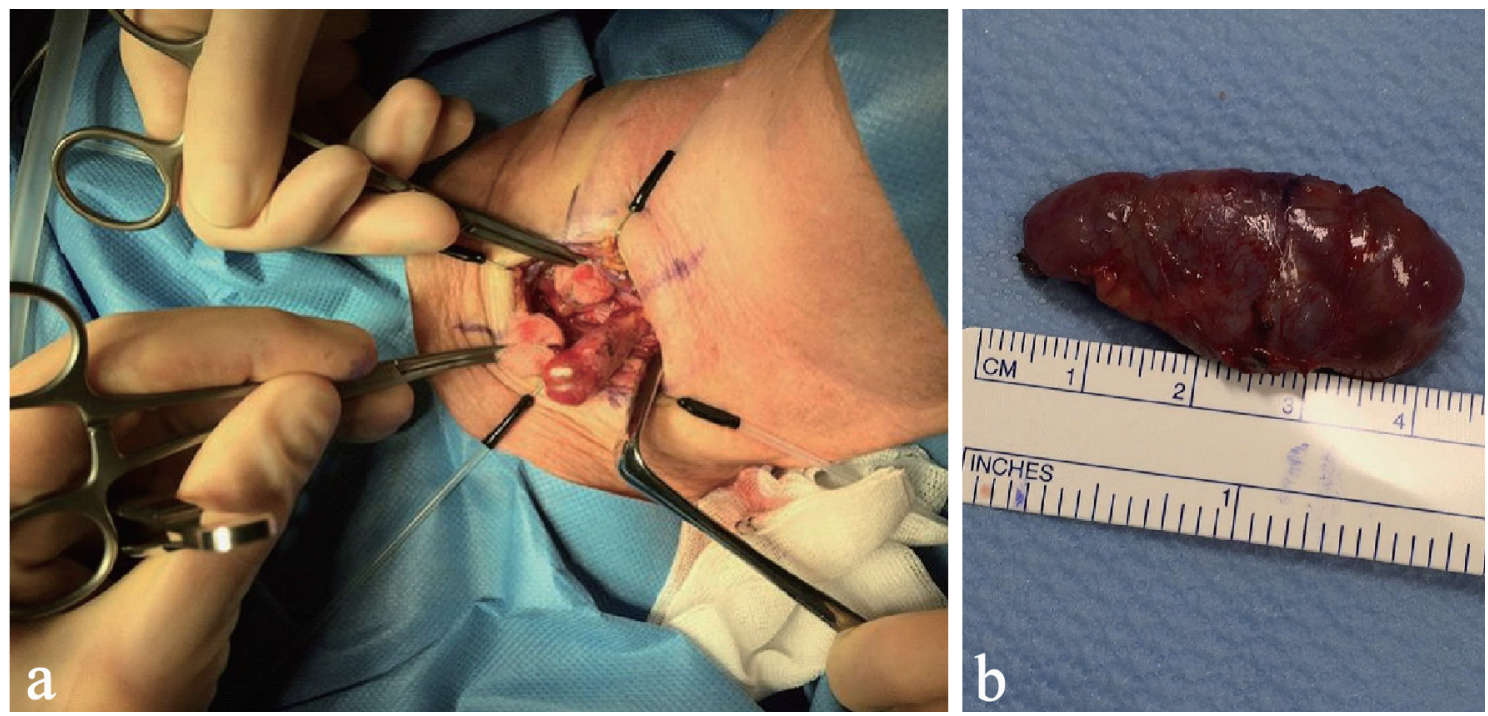

Figure 5. Per-operative image of the PTA (a) and postoperative image showing the adenoma of $4.9 \mathrm{~g}$ (b). PTA: parathyroid adenoma.
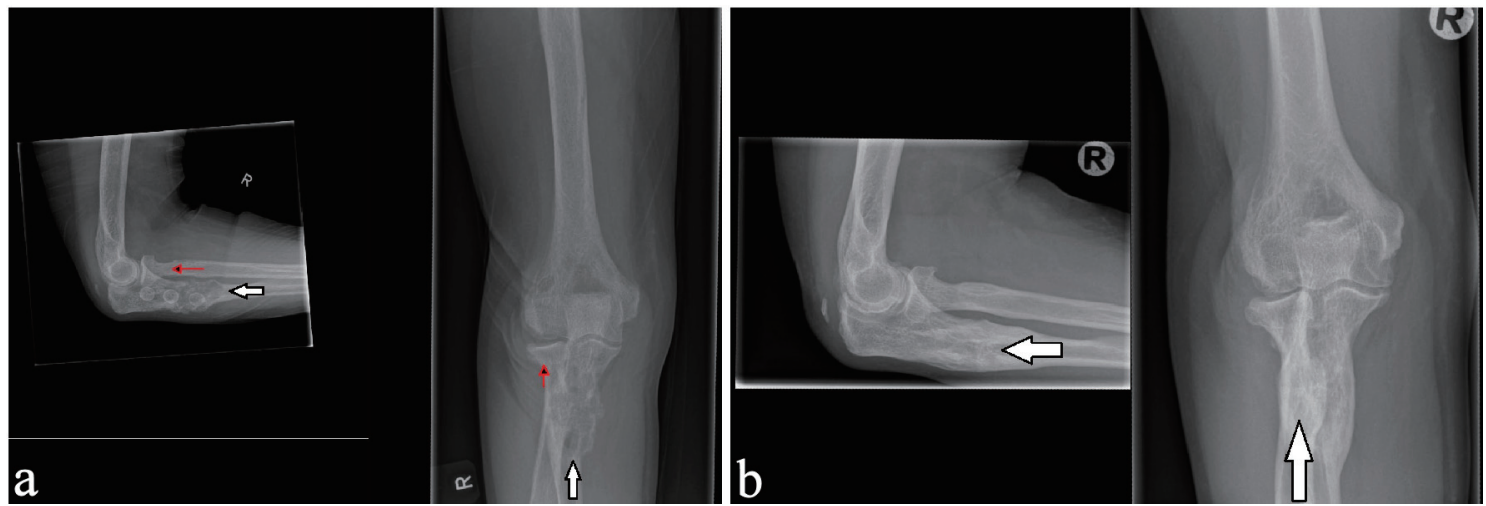

Figure 6. X-ray examinations after the fall trauma (a) and 14 months afterwards (b) showing regression of the osteolytic lesion in the ulna. 

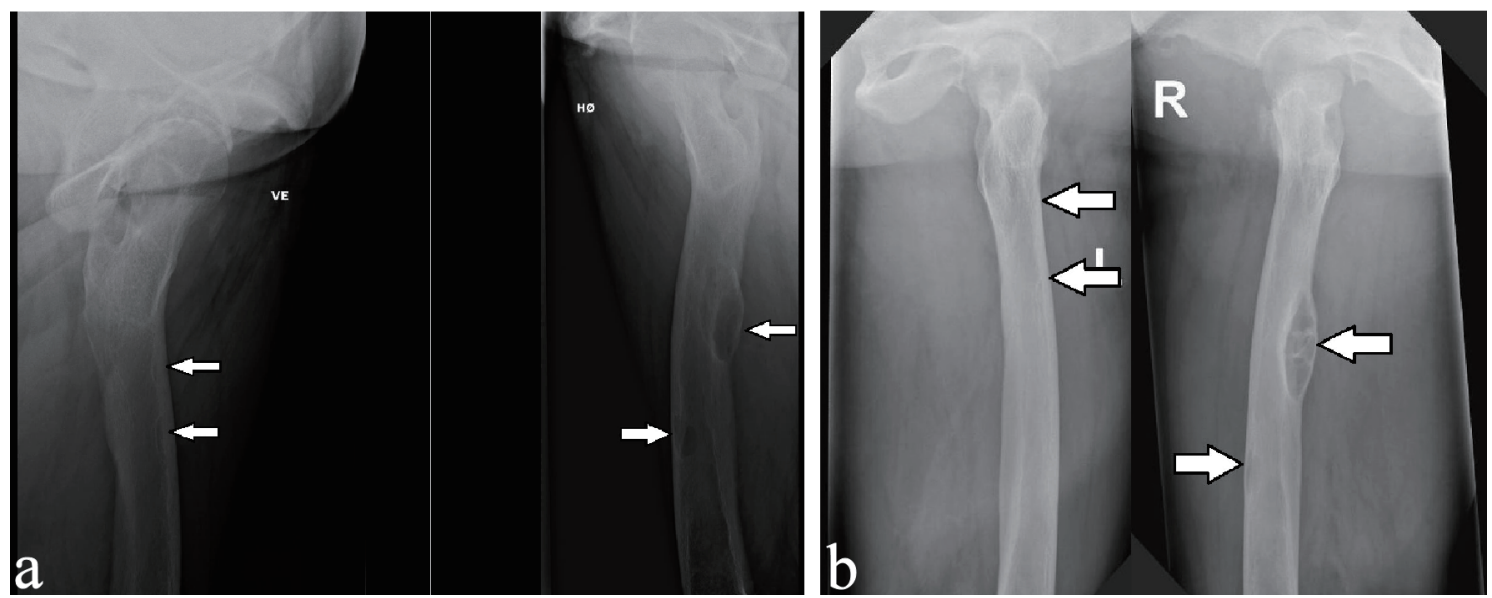

Figure 7. X-ray examinations after the fall trauma (a) and 14 months afterwards (b) showing regression of the osteolytic lesions in both femurs.

ity leading to a considerable increase in calcium uptake in the bones frequently resulting in hypocalcemia, and sometimes "the HBS" $[2,19,20]$. HBS is a severe, sudden and prolonged hypocalcemia following parathyroidectomy in patients with elevated PTH levels preoperatively. The incidence is $12-20 \%$ among PHPT patients $[2,19,20]$. In our case, the PTH level declined by $72 \%, 10$ min after removal of the adenoma (369 $\mathrm{pmol} / \mathrm{L}$ preoperatively, and $56 \mathrm{pmol} / \mathrm{L}$ postoperatively). The development of HBS in our patient was probably influenced by the presence of several risk factors, such as female gender, age $>50$ years, widespread brown tumors, elevated preoperative levels of PTH, urea, alkaline phosphatase, and large PTA [2]. Our patient received long-term active vitamin D along with calcium carbonate, vitamin $\mathrm{D}$, and magnesium upon discharge. Other reported cases of brown tumors had received similar treatment $[4,6,8,19,21]$.

The brown tumors may completely regress after the normalisation of PTH level. Thus, no supplemental orthopaedic
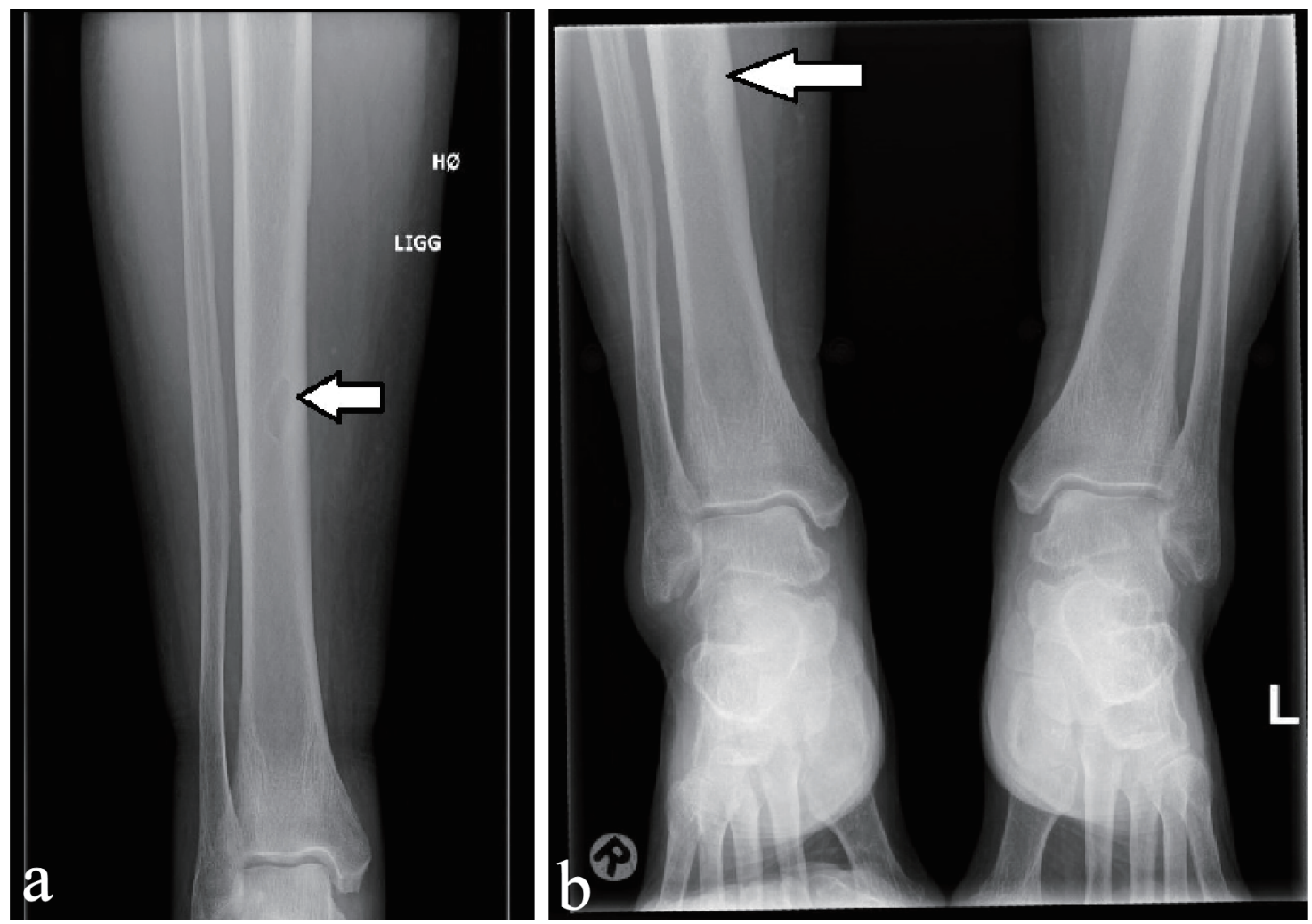

Figure 8. X-ray examinations after the fall trauma (a) and 14 months afterwards (b) showing regression of the osteolytic lesion in the tibia. 
surgery will be needed $[1,4,6,13,18]$. Regression of brown tumors and a $35-131 \%$ gain in bone density in the lumbar spine and proximal femur have been reported just 1 year after parathyroidectomy $[4,6,11,19]$. In our case, the osteoporosis regressed, and the osteolytic lesions were almost unrecognizable about 14 months after parathyroidectomy. It is important to consider brown tumors as differential diagnosis for osteolytic bone lesions. Two cases of misdiagnosed brown tumors were reported [14]. In both cases, patients were believed to have multiple osteolytic bone metastases and received palliative chemo- and radiotherapy. Both patients were later on diagnosed with PHPT after the blood tests showed elevated levels of PTH and calcium [14]. Another study reported a patient with a misdiagnosed brown tumor in the forearm resembled an osteolytic metastasis. The patient underwent resection of 9.5 $\mathrm{cm}$ of the distal ulna. The blood tests showed elevated PTH and calcium levels 2 weeks postoperatively. The patient was, finally, diagnosed with parathyroid carcinoma, and the resection of the brown tumor in the ulna was therefore unnecessary [4].

In conclusion, it is essential to remember that not all osteolytic bone lesions are malignant. Brown tumors should be considered as a differential diagnosis when multiple osteolytic lesions are discovered incidentally, despite their rare occurrence to avoid unnecessary treatment and/or intervention. Our case also confirms that brown tumors are reversible, even when the lesions are multiple and have invasive characteristics, and that removal of the PTA should not be delayed.

\section{Acknowledgments}

None to declare.

\section{Financial Disclosure}

The study did not receive any form of financial support.

\section{Conflict of Interest}

None to declare.

\section{Informed Consent}

The patient has given an informed consent as required by our ethics committee.

\section{Author Contributions}

$\mathrm{PA}$ and $\mathrm{ZH}$ contributed to the study concept and design, manuscript development and writing. PA, IH, WT, ZH, and LT contributed to manuscript concept and design, acquisition of images. PA, ZH, LT, PE, WT and IH contributed to manuscript authorization.

\section{Data Availability}

Any inquiries regarding supporting data availability of this study should be directed to the corresponding author.

\section{References}

1. Dagang DJ, Gutierrez JB, Sandoval MA, Lantion-Ang FL. Multiple brown tumours from parathyroid carcinoma. BMJ Case Rep. 2016;2016.

2. Ghilardi G, De Pasquale L. Hungry bone syndrome after parathyroidectomy for primary hyperthyroidism. Surgery Curr Res. 2014;4(2):1000168.

3. Madkhali T, Alhefdhi A, Chen H, Elfenbein D. Primary hyperparathyroidism. Ulus Cerrahi Derg. 2016;32(1):5866.

4. Panagopoulos A, Tatani I, Kourea HP, Kokkalis ZT, Panagopoulos K, Megas P. Osteolytic lesions (brown tumors) of primary hyperparathyroidism misdiagnosed as multifocal giant cell tumor of the distal ulna and radius: a case report. J Med Case Rep. 2018;12(1):176.

5. Radulescu D, Chis B, Donca V, Munteanu V. Brown tumors of the femur and pelvis secondary to a parathyroid carcinoma: report of one case. Rev Med Chil. 2014;142(7):919-923.

6. Brabyn P, Capote A, Belloti M, Zylberberg I. Hyperparathyroidism diagnosed due to brown tumors of the jaw: a case report and literature review. J Oral Maxillofac Surg. 2017;75(10):2162-2169.

7. Alfawareh MD, Halawani MM, Attia WI, Almusrea KN. Brown tumor of the cervical spines: a case report with literature review. Asian Spine J. 2015;9(1):110-120.

8. Gadhia T, Adegun OK, Fortune F. Brown tumours: widespread involvement of multiple maxillofacial bones and cervical spine. BMJ Case Rep. 2014;2014.

9. Xie C, Tsakok M, Taylor N, Partington K. Imaging of brown tumours: a pictorial review. Insights Imaging. 2019;10(1):75.

10. Khalatbari MR, Moharamzad Y. Brown tumor of the spine in patients with primary hyperparathyroidism. Spine (Phila Pa 1976). 2014;39(18):E1073-1079.

11. Hu J, He S, Yang J, Ye C, Yang X, Xiao J. Management of brown tumor of spine with primary hyperparathyroidism: A case report and literature review. Medicine (Baltimore). 2019;98(14):e15007.

12. Zou H, Song L, Jia M, Wang L, Sun Y. Brown tumor of multiple facial bones associated with primary hyperparathyroidism: A clinical case report. Medicine (Baltimore). 2018;97(33):e11877.

13. Minisola S, Gianotti L, Bhadada S, Silverberg SJ. Classical complications of primary hyperparathyroidism. Best Pract Res Clin Endocrinol Metab. 2018;32(6):791-803.

14. Misiorowski W, Czajka-Oraniec I, Kochman M, Zgliczynski W, Bilezikian JP. Osteitis fibrosa cystica-a forgotten radiological feature of primary hyperparathyroidism. Endocrine. 2017;58(2):380-385.

15. Jervis L, James M, Howe W, Richards S. Osteolytic le- 
sions: osteitis fibrosa cystica in the setting of severe primary hyperparathyroidism. BMJ Case Rep. 2017;2017.

16. Garla VV, Akhtar I, Salim S, Subauste A. Osteitis fibrosa cystica masquerading as bone neoplasm. BMJ Case Rep. 2018;2018.

17. Agnihotri M, Kothari K, Naik L. Omega Brown tumor of hyperparathyroidism. Diagn Cytopathol. 2017;45(1):4344.

18. Can O, Boynuegri B, Gokce AM, Ozdemir E, Ferhatoglu F, Canbakan M, Sahin GM, et al. Brown tumors: a case report and review of the literature. Case Rep Nephrol Dial. 2016;6(1):46-52.
19. Anwar F, Abraham J, Nakshabandi A, Lee E. Treatment of hypocalcemia in hungry bone syndrome: A case report. Int J Surg Case Rep. 2018;51:335-339.

20. Jakubauskas M, Beisa V, Strupas K. Risk factors of developing the hungry bone syndrome after parathyroidectomy for primary hyperparathyroidism. Acta Med Litu. 2018;25(1):45-51.

21. Radu CP, Daniealopol V, Santini A, Darie R, Sala DT. Fatal hypocalcaemia due to hungry bone syndrome with secondary refractory hyperparathyroidism after parathyroidectomy: a case report. J Crit Care Med (Targu Mures). 2019;5(4):140-144. 